\title{
DIFERENTES NÍVEIS DE ÁCIDO FÓLICO E NICOTÍNICO EM DIETAS PARA FRANGOS DE CORTE
}

\author{
DIFFERENT LEVELS OF FOLIC AND NICOTINIC \\ ACID IN BROILER'S DIET
}

\author{
Elizabeth Santin ${ }^{1}$ Norberto Luiz Ahrens ${ }^{2}$ Irineo Zanella ${ }^{3}$ \\ Paulo Tabajara Chaves Costa ${ }^{4}$ Alex Maiorka $^{5}$ \\ Leandro Magon ${ }^{5}$
}

RESUMO

\begin{abstract}
O experimento teve como objetivo avaliar níveis de ácido fólico e nicotínico sobre o desempenho de frangos de corte. Foram utilizados 400 pintos de corte de l dia de idade, machos, da linhagem Hubbard em um delineamento experimental inteiramente casualisado em esquema fatorial $2 \times 2$, com 2 vitaminas (ácido fólico e ácido nicotínico), 2 níveis (alto e baixo), sendo cada unidade experimental constituída de 20 aves. Os níveis utilizados das vitaminas ácido fólico $(A F)$ e ácido nicotínico (AN) foram oriundos da média utilizada pelas grandes integrações avícolas do sul do Brasil, sendo o nível denominado alto igual a $120 \%$ da média das empresas e o nível baixo igual a $80 \%$ da média. As dietas foram calculadas para serem isonutritivas, variando apenas as vitaminas em estudo. No término do período experimental, foram colhidos dados para análise dos seguintes parâmetros: consumo de ração, ganho médio de peso, conversão alimentar e viabilidade criatória. Também se avaliaram dados de índice de eficiência produtiva, rendimento de carcaça, gordura abdominal e peso de fígado. Os resultados sugerem que o s níveis considerados baixos não acarretam prejuízo no que se refere aos parâmetros zootécnicos analisados.
\end{abstract}

Palavras-chave: frangos de corte, desempenho, ácido fólico, ácido nicotínico, vitaminas.

\section{SUMMARY}

The objective of lhe experiment was to evaluate the performance of broiler chickens in different leveis of acid folie ana nicotinic. A total number of 400 one-day-old male chicks, of

\begin{abstract}
Hubbard Une were used in a design completely randomized factorial $2 \times 2$, with two vitamins (folie and nicotinic acid) and two leveis (high and tow), being each experimental unit composed 0/20 birds. The vitamins leveis used are average leveis used by the most important raising poultry industry in the southem of Brazil. The vitamins used were defined in two leveis, low (80\% of médium levei) and high (120\% of médium levei). The fed diets were formulated to be isonutritives, changing onty the studied vitamins. At the end ofthe period, the foüawing parameters were evaluated: feed intake, body weight gain, feed conversion, productive efficiency Índex, liver weight, abdominal fat weight. and carcass yield. No statistical diferences were found in the parameters observed. The results found at the end of the experiment suggested that low leveis could be used, without performance damages.
\end{abstract}

Key words: broiler, performance, folie acid, nicotinic acid, vitamins.

\section{INTRODUÇÃO}

As vitaminas desempenham funções essenciais no metabolismo fisiológico dos animais, e elas podem ficar comprometidas quando houver insuficiência, (BELL, 1989). Em geral, as aves não podem sintetizar vitaminas, as quais devem ser suplementadas na dieta, em níveis suficientes para suprir suas necessidades, principalmente, quando

\footnotetext{
${ }^{1}$ Acadêmico da Universidade Federal de Santa Maria (UFSM), Bolsista Iniciação Científica - CNPq.

${ }^{2}$ Zootecnista, Pós-Graduação Zootecnia, UFSM.

${ }^{3}$ Professor Adjunto, Departamento de Zootecnia UFSM, 97105-900, Santa Maria - RS. E-mail: izanella@ccr.ufsm.br. Autor para correspondência.

${ }^{4}$ Médico Veterinário, Pesquisador $\mathrm{CNPq}$

${ }^{5}$ Acadêmico de Zootecnia e Veterinária, UFSM. 
ocorrer variações nos fatores genéticos, ambientais e sanitários (LARBIER \& LECLERCQ, 1992). O Ácido fólico é essencial ao metabolismo dos compostos monocarbonados, além de ser cosubstrato para enzimas em muitas reações no metabolismo dos aminoácidos e nucleotídeos. Sua deficiência causa uma diminuição na biossíntese de DNA e RNA levando a uma redução no número de células em divisão, o que resulta em inibição do crescimento, diminuição do apetite, penas eriçadas, dermatite, anemia, diarreia branca e má formação do bico das aves (COMBS, 1992). PESTY \& ROWLAND (1991) compararam dietas com e sem adição de ácido fólico para aves, e concluíram que de $\mathrm{O}$ a 18 dias o crescimento dos frangos aumentou significativamente $(\mathrm{P}<0,05)$, com a suplementação $\mathrm{de} 1,52 \mathrm{mg} / \mathrm{kg}$ de folacina.

Quase todas as espécies animais parecem ter capacidade, em diferentes graus, para sintetizar o ácido nicotínico e seus principais produtos, o NADH e o NADPH, a partir do aminoácido essencial triptofano, no entanto, em geral, essa conversão é um processo pouco eficiente. As aves necessitam, em média, $45 \mathrm{~g}$ de triptofano para produzir $\lg$ de niacina (COMBS, 1992). Como o NADH e o NADPH são essenciais na produção de ATP para a cadeia respiratória, sua deficiência pode levar a um déficit energético, podendo acarretar a morte do organismo. Para comparar a inter-relação entre o triptofano e a niacina, SAHARASINGHE \& WENK (1990) forneceram a frangos de corte suplementação com triptofano na dieta, combinado ou não com niacina, e observaram que as aves que receberam triptofano associado à niacina na dieta apresentaram maior ganho de peso e consumo alimentar, quando comparadas com os outros grupos, sugerindo que é importante que essa vitamina seja fornecida na ração. No entanto, RUIZ \& HARMS (1990), ao suplementarem dietas de frangos de corte (3-7 semanas de idade) com níveis crescentes de ácido nicotínico (O a $36 \mathrm{mg} / \mathrm{kg}$ ), não observaram qualquer influência sobre o desempenho das aves. Os referidos autores concluíram que uma dieta à base de milho e soja, contendo $22 \mathrm{mg}$ de ácido nicotínico por $\mathrm{kg}$ e $0,23 \%$ de triptofano, foi adequada para o máximo crescimento e eficiência alimentar de frangos de corte entre 3 e 7 semanas de idade.

A suplementação de vitaminas na dieta representa, em média, $0,2 \%$ do volume total da ração, aumentando o custo da criação em aproximadamente $2 \%$, o que faz com que se busquem alternativas para diminuir os níveis utilizados sem que haja prejuízos no desempenho final de frangos de corte. No Brasil, os padrões de recomendações e os níveis utilizados baseiam-se em literatura estrangeira, orientações dos próprio fabricantes, manuais de linhagens e experiência das empresas.

Talvez estas variações nas respostas aos diferentes níveis vitamínicos estudados sugiram quehá a necessidade de novas adaptações nas fórmulas dos premixes de acordo com as alterações do meio ambiente e do organismo animal em que estamos trabalhando, pois, segundo FERKET \& QURESHI (1992), apesar das exigências para vitaminas estarem definidas para aves, os níveis de vitaminas nas rações comerciais geralmente são mais altos que as recomendações do NRC (1984), e assim, melhoram a sobrevivência das aves. No entanto, torna-se importante destacar que níveis muito elevados de vitaminas nas dietas podem acarretar prejuízos económicos, por aumentar o custo de produção, porém, também se faz necessário lembrar que, de acordo com XU et al. (1989), a limitação das vitaminas totais da dieta em $75 \%$ dos níveis exigidos pelo NRC (1984) resultaram em aumento da mortalidade e depressão na imunidade de frangos de corte.

RUTZ et al. (1999) apresentaram os níveis vitamínicos utilizados no premix em dietas para frangos de corte, durante a fase final, pelas principais integrações avícolas do sul do Brasil e recomendações internacionais. Com base nos resultados publicados, observa-se a grande variação nos níveis vitamínicos utilizados entre as empresas avícolas. Dessa forma, é de grande interesse que novos estudos sejam conduzidos para determinar níveis que proporcionem melhor retorno económico, sem que haja prejuízo ao desempenho das aves.

Com o objetivo de avaliar diferentes níveis das vitaminas ácido fólico e ácido nicotínico nas dietas $(20 \%$ superior e $20 \%$ inferior aos níveis de premixes utilizados pelas integrações avícolas do sul do Brasil) sobre o desempenho de frangos de corte foi conduzido o presente trabalho.

\section{MATERIAL E MÉTODOS}

O experimento foi desenvolvido no Setor de Avicultura da Universidade Federal de Santa Maria (UFSM), Santa Maria, RS, em um aviário convencional para frangos de corte, dividido em 20 boxes telados, com dimensões de 1,5 x 1,5m, os quais foram preparados com cama de maravalha $(10 \mathrm{~cm})$, uma campânula elétrica com lâmpada incandescente de 100 watts, um bebedouro pendular automático e um comedouro tipo bandeja, que foi substituído no $7^{\mathrm{a}}$ dia por um comedouro tubular( $15 \mathrm{~kg})$. O período experimental totalizou 48 dias, dividido em 3 períodos: inicial (1-21 dias), 
crescimento (22-42 dias) e final (43-48 dias), sendo utilizados 400 pintos de corte, machos, da linhagem Hubbard. No término de cada período, todas as aves foram pesadas, bem como a sobra da ração para calcular o consumo alimentar. No $48^{\circ}$ dia de idade, $50 \%$ dos frangos de cada unidade experimental foram abatidos para avaliação do rendimento de carcaça, gordura abdominal e peso de fígado, sendo selecionadas as aves que apresentassem o peso médio a respectiva repetição.

As vitaminas sintéticas foram obtidas em distribuidoras comerciais da região, assegurando a idoneidade do produto; foram pesadas em balança elétrica com precisão de $0,001 \mathrm{~g}$, pré-misturadas com farinha de milho em misturador tipo "Y", com capacidade de $30 \mathrm{~kg}$ e $38 \mathrm{rpm}$ por um período de 10 minutos. O premix vitamínico, excetuando as vitaminas em estudo, adicionado de cloreto de colina e DL-metionina foi utilizado nas mesmas quantidades para todas as combinações, decrescentes conforme os períodos: inicial (100\%), crescimento $(83,33 \%)$ e final $(66,66 \%)$, conforme tabela 1 .

Os níveis utilizados de ácido fólico e ácido nicotínico foram originados a partir da média utilizada pelas integrações avícolas do sul do Brasil. Considerando-se como nível baixo aquele contendo $80 \%$ da concentração média das empresas para essas vitaminas, e sendo denominado de nível alto quando a quantidade para essas substâncias na dieta representava $120 \%$ da concentração média utilizada pelas empresas, conforme apresentado na tabela 2 . As dietas apresentavam-se sob a forma farelada e foram formuladas à base de milho e farelo de soja, sendo que a proporção dos ingredientes utilizados nas

Tabela 1 - Níveis vitamínicos utilizados por $\mathrm{kg}$ da dieta nos períodos inicial, crescimento e final, excetuando as vitaminas em estudo.

\begin{tabular}{ccccc}
\hline & & & & \\
& & & \\
& & & \\
Vitaminas & Unidade & Inicial & Crescimento & Final \\
& & & & \\
\hline & & & & \\
$\mathrm{A}$ & $\mathrm{UI}$ & 11000 & 9166 & 7333 \\
$\mathrm{D}_{3}$ & $\mathrm{UI}$ & 2475 & 2063 & 1650 \\
$\mathrm{E}$ & $\mathrm{mg}$ & 21,25 & 17,70 & 14,16 \\
$\mathrm{~K}_{3}$ & $\mathrm{mg}$ & 3,43 & 2,86 & 2,29 \\
$\mathrm{~B}_{1}$ & $\mathrm{mg}$ & 2,50 & 2,08 & 1,66 \\
$\mathrm{~B}_{2}$ & $\mathrm{mg}$ & 5,00 & 4,17 & 3,33 \\
$\mathrm{~B}_{6}$ & $\mathrm{mg}$ & 3,75 & 3,12 & 2,50 \\
$\mathrm{~B}_{12}$ & $\mathrm{mcg}$ & 16,54 & 13,54 & 10,83 \\
Biotina $_{12}$ & $\mathrm{mg}$ & 0,19 & 0,16 & 0,12 \\
CL-Colina & $\mathrm{g}$ & 0,80 & 0,67 & 0,53 \\
& & & & \\
\hline
\end{tabular}

Tabela 2 - Níveis das duas vitaminas-ácidas (ácido fólico e ácido nicotínico) por período de criação em $\mathrm{mg} / \mathrm{kg}$ da dieta.

\begin{tabular}{|c|c|c|c|c|c|c|}
\hline \multirow[b]{3}{*}{ Vitaminas } & \multicolumn{6}{|c|}{ Períodos } \\
\hline & \multicolumn{2}{|c|}{ Inicial } & \multicolumn{2}{|c|}{ Crescimento } & \multicolumn{2}{|c|}{ Final } \\
\hline & Alto $(\uparrow)$ & Baixo $(\downarrow)$ & Alto & Baixo & Alto & Baixo \\
\hline $\begin{array}{l}\text { Ácido Fólico (AF) } \\
\text { Ácido Nicotínico (AN) }\end{array}$ & $\begin{array}{c}1,2 \\
22,5\end{array}$ & $\begin{array}{c}0,8 \\
15,0\end{array}$ & $\begin{array}{c}1,00 \\
18,75\end{array}$ & $\begin{array}{c}0,66 \\
12,50\end{array}$ & $\begin{array}{c}0,80 \\
15,00\end{array}$ & $\begin{array}{l}0,53 \\
9,99\end{array}$ \\
\hline
\end{tabular}

rações e suas composições calculadas encontram-se na tabela 3. Foram avaliados os seguintes parâmetros: consumo de ração, ganho de peso, conversão alimentar, viabilidade criatória, índice de eficiência produtiva, rendimento de carcaça, peso de fígado e gordura abdominal.

O delineamento experimental utilizado foi o inteiramente casualizado em esquema fatorial (2x2) constituído de duas vitaminas (ácido fólico e ácido nicotínico) e dois níveis vitamínicos (alto e

Tabela 3 - Proporção dos ingredientes e composição calculada das dietas experimentais de acordo com as fases criátorias.

\begin{tabular}{|c|c|c|c|}
\hline & \multicolumn{3}{|c|}{ Períodos } \\
\hline Ingredientes (\%) & Inicial & Crescimento & Final \\
\hline Milho & 51,50 & 59,34 & 67,11 \\
\hline$F^{o}$ de Soja & 39,60 & 32,50 & 26,10 \\
\hline Óleo Vegetal & 5,11 & 4,48 & 3,64 \\
\hline Fosfato Bicálcico & 1,72 & 1,81 & 1,13 \\
\hline $\mathrm{F}^{\mathrm{a}}$ de Ostra & 1,24 & 1,13 & 1,37 \\
\hline Sal & 0,30 & 0,30 & 0,30 \\
\hline DL-Metionina & 0,15 & 0,12 & 0,10 \\
\hline Cloreto de Colina & 0,08 & 0,06 & 0,05 \\
\hline Coccidicida & 0,10 & 0,05 & - \\
\hline Px Mineral* & 0,10 & 0,10 & 0,10 \\
\hline Px Vitamínico & 0,10 & 0,10 & 0,10 \\
\hline
\end{tabular}

Composição Calculada

\begin{tabular}{lccc}
\hline & & & \\
PB (\%) & 21,50 & 19,50 & 17,50 \\
EM (kcal/kg) & 3050 & 3100 & 3150 \\
Ca (\%) & 0,95 & 0,90 & 0,85 \\
Fósforo Disponível(\%) & 0,42 & 0,44 & 0,33 \\
Metionina & 0,45 & 0,42 & 0,38 \\
Lisina (\%) & 1,14 & 1,03 & 0,92 \\
& & & \\
\hline
\end{tabular}

*Composição por kg da dieta.

$\mathrm{Fe}=65 \mathrm{mg} ; \mathrm{Cu}=10 \mathrm{mg} ; \mathrm{Zn}=55 \mathrm{mg} ; \mathrm{I}=0,50 \mathrm{mg} ; \mathrm{Se}=0,15$ $\mathrm{mg} ; \mathrm{Mn}=0,70 \mathrm{mg}$.

Ciência Rural, v. 30, n. 4, 2000. 
baixo) e 5 repetições, sendo cada unidade experimental constituída por 20 aves. As análises estatísticas foram realizadas através do programa SÃS (1990). Os dados obtidos foram submetidos à análise de variância e nos parâmetros que acusaram diferenças estatísticas significativas, em nível de $5 \%$, as médias foram comparadas pelo teste de Tukey.

\section{RESULTADOS E DISCUSSÃO}

Os dados de consumo de ração, ganho de peso, conversão alimentar do período total e viabilidade criatória encontram-se na tabela 4. Os resultados indicaram que esses parâmetros não foram influenciados significativamente $(\mathrm{P}>0,05)$ pêlos níveis suplementares das vitaminas avaliadas, os quais concordam com o mencionado por RUIZ \& HARMS (1990), que utilizando diferentes níveis de suplementação de ácido nicotínico para frangos de corte, também não observaram diferenças significativas. No entanto, os resultados do presente experimento diferem dos encontrados por ALNASSER et al. (1986) que testaram níveis adicionais de $25 \%$ a $50 \%$ das vitaminas recomendadas pelo NRC (1984) e concluíram que os níveis propostos para frangos de corte deveriam ser aumentados para proporcionar um maior consumo alimentar.

Os dados de índice de eficiência produtiva, rendimento de carcaça, peso de fígado e gordura abdominal, determinados com 48 dias de idade, encontram-se na tabela 5, e também não apresentam diferenças estatísticas significativas $(\mathrm{P}>0,05)$. Esses resultados concordam com o que foi encontrado por RUBIN (1989) que, testando diferentes níveis de suplementação (75 a $130 \%$ do premix padrão) em frangos de corte não encontrou diferença significativa

Tabela 4 - Resultados médios de consumo de ração (CR), ganho de peso (GP), conversão alimentar (CA) e viabilidade criatória (VC) no período de $1-48$ dias de idade para os diferentes tratamentos experimentais.

\begin{tabular}{|c|c|c|c|c|}
\hline Tratamentos & C R (kg) & G P (kg) & $\mathrm{C} \mathrm{A}(\mathrm{kg} / \mathrm{kg})$ & $\mathrm{VC}(\%)$ \\
\hline $\mathrm{T} 1-\mathrm{AF}(\uparrow)$ & 4,75 & 2,30 & 2,07 & 95,00 \\
\hline $\mathrm{T} 2-\mathrm{AF}(\downarrow)$ & 4,73 & 2,31 & 2,05 & 96,25 \\
\hline T3-AN ( & 4,76 & 2,25 & 2,12 & 95,63 \\
\hline 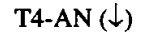 & 4,69 & 2,23 & 2,10 & 95,60 \\
\hline Média & 4,73 & 2,27 & 2,08 & 95,62 \\
\hline $\mathrm{CV}(\%)$ & 3,24 & 3,77 & 3,36 & 3,53 \\
\hline
\end{tabular}

$(\mathrm{P}>0,05)$.
Tabela 5 - Resultados médios de índice de eficiência produtiva (IEP), rendimento de carcaça (RC), peso de fígado (PF) e gordura abdominal (GA) aos 48 dias de idade, para os diferentes tratamentos experimentais.

\begin{tabular}{crrrr}
\hline Tratamentos & IEP & RC $(\%)$ & PF(g) & GA (g) \\
\hline T1-AF $(\uparrow)$ & 253,36 & 75,05 & 51,43 & 35,18 \\
T2-AF $(\downarrow)$ & 234,10 & 76,86 & 51,62 & 27,68 \\
T3-AN $\uparrow$ ( $)$ & 242,47 & 73,74 & 46,19 & 23,06 \\
T4-AN $(\downarrow)$ & 244,99 & 76,72 & 47,18 & 26,06 \\
Média & 243,73 & 75,59 & 49,10 & 27,99 \\
CV $(\%)$ & 6,22 & 5,26 & 9,17 & 13,08 \\
\hline
\end{tabular}

$(P>0,05)$.

para esses parâmetros. Os resultados para peso de fígado observados no presente experimento, discordam de PESTY \& ROWLAND (1991) que observaram uma redução significativa $(P<0,05)$ no peso de fígado de aves suplementadas com $1,52 \mathrm{mg} / \mathrm{kg}$ de ác. fólico até o $18^{\circ}$ dia de vida, em relação as não suplementadas com esta vitamina.

Considerando-se que os parâmetros produtivos não foram afetados pêlos níveis vitamínicos avaliados, isso denota que mesmo a redução de $20 \%$ dos níveis das vitaminas em estudo não causaram efeitos que pudessem afetar o bom desempenho das aves, o que indica que os níveis dessas vitaminas podem ser reduzidos até esse nível nas dietas para frangos de corte.

\section{CONCLUSÕES}

Nas condições em que se realizou o presente estudo, conclui-se que os parâmetros avaliados não são influenciados pelo acréscimo ou diminuição em $20 \%$ nos níveis das vitaminas ácido fólico e nicotínico no premix vitamínico em relação aos níveis médios utilizados pelas integrações avícolas do sul do Brasil.

\section{REFERÊNCIAS BIBLIOGRÁFICAS}

AL-NASSER, A.Y., AL-AWADI, A.A., DIAS, M.F., et al The effect of adding essential amino acids and vitamins to the rations ofbroilers. Poult Sei, v.65, p.742-748,1986.

BELL, E.P. Upper limit of vitamin E in infant formulas. J Nutr, v.119,p.1829-1831. 1989.

COMBS Jr., G.F. The vitamins. Fundamental aspects in nutrition and health San Diego: Academic, 1992. 595p.

FERKET, P.R, QURESHI, M.A. Performance and immunity of heat-stressed broilers fed vitamin- and electrolytesuppiemented drinking water. Poult Sei, v.71, p.88-97, 1992. 
LARBIER, M., LECLERCQ, B. Nutrition and feeding of poultry. Loughborough : Notüngham University, 1992. 305p.

NATIONAL RESEARCH COUNCIL (NRC). Nutrient requirements of domestic animais. Nutrient requirements of poultry. Washington : National Academy, 1984. 71p.

PESTY, G.M., ROWLAND, G.N.I. Folate deficiency in chicks fed diets containing practical ingrediente. Poult Sei, v.70, p.600-604, 1991

RUBIN, M.A. Efeitos dos níveis vitaniínicos sobre o desempenho de frangos de corte em duas estações (inverno e verão). Santa Maria, RS, 1989. 98p. Dissertação (Mestrado em Zootecnia) - Curso de Pós-graduação em Zootecnia, Universidade Federal de Santa Maria, 1989.

RUIZ, N., HARMS, R.H. The lack of response of broiler chickens to suppiemental niacin when fed a com-soybean meai diet from 3 to 7 weeks of age. Poult Sei, v.69, p.2231-2234,1990.
RUTZ, F., XAVIER, E.G., DADALT, G.M. Exigências nutricionais para a fase final (energia, aminoácidos, vitaminas, minerais e aditivos). In: CONFERÊNCIA APINCO'99 DE CIÊNCIA E TECNOLOGIA AVÍCOLAS, 1999, Campinas, SP. Anais... Campinas ; FACTA, 1999. p.29-54.

SAHARASINGHE, K., WENK, C. Interelationship between tryptophan and niacin in the nutrition of growing chickens. Nutrition Abstracts and Review, v.60, p. 1007,1990.

SÃS.- Instituto SÃS® User's Guide: Statistics. Version 5. Edition. Cary, NC : SÃS Instituto, 1990. 956p.

XU, T., COOK, M.E., BLAKE, J.P. Vitamin suppiementation on performance and immune response of turkeys. Poult Sei, v.68,p.161,1989.

Ciência Rural, v. 30, n. 4, 2000. 